\title{
A randomized trial of aprotinin (Trasylol) on blood loss, blood product requirement, and myocardial injury in total arterial grafting
}

David P. Taggart, MD (Hons), PhD, FRCS

Veronica Djapardy, BM, BCh

Madhav Naik, MB

Amanda Davies, RCN

See related articles on pages 944 and 1061.
From the Oxford Heart Centre, John Radcliffe Hospital, Oxford, United Kingdom.

Bayer Pharmaceuticals funded the study, performed statistical analyses of the data, and provided the Medical Research Report.

Received for publication May 21, 2002; revisions requested Aug 15, 2002; revisions received Nov 12, 2002; accepted for publication Feb 3, 2003.

Address for reprints: David P. Taggart, Oxford Heart Centre, John Radcliffe Hospital, Oxford OX3 9DU, United Kingdom (Email: david.taggart@orh.nhs.uk).

J Thorac Cardiovasc Surg 2003;126: 1087-94

Copyright $(\underset{0}{ } 2003$ by The American Association for Thoracic Surgery

$0022-5223 / 2003 \$ 30.00+0$

doi:10.1016/S0022-5223(03)00961-9
Background: Total arterial grafting is increasingly preferred in coronary artery bypass grafting, but it increases blood loss. Aprotinin (Trasylol; Bayer Corp, Leverkusen, Germany) reduces blood loss in cardiac surgery but has not been subjected to a randomized trial in total arterial grafting.

Methods: A single-center, randomized, double blind, placebo-controlled trial of aprotinin administration in total arterial grafting was performed. The primary outcome variable was postoperative blood loss, and the secondary outcome variable was the number of units of donor blood or coagulant products transfused. The incidence of myocardial injury was determined from serial measurements of cardiac troponin $\mathrm{T}$ and creatine kinase-MB and renal injury from serum creatinine.

Results: The placebo group $(\mathrm{n}=34)$ and aprotinin group $(\mathrm{n}=36)$ were similar with respect to all preoperative and intraoperative comparisons. One patient in each group underwent reexploration for bleeding. Open-label aprotinin was administered to 9 patients in the placebo group (26\%) and to 2 patients in the aprotinin group $(6 \%)$. There was a highly significant reduction in the median (interquartile range) blood loss in the aprotinin group compared with the placebo group $(785 \mathrm{~mL}$ [590-1025 mL] vs $1525 \mathrm{~mL}$ [1175-1920 mL], respectively). Similarly, the aprotinin group demonstrated a marked reduction in the need for blood transfusion (77\% vs $39 \% ; P=.0001)$, the mean number of transfused blood units $(2.6$ vs $0.8, P<.001)$, and the number of patients requiring coagulant products $(24 \%$ vs $3 \% ; P<.001)$. There was no difference in myocardial injury in the 2 groups. Four patients in the aprotinin group had persistently elevated creatinine levels in the postoperative period ( 3 of whom had elevated preoperative creatinine levels and perioperative complications).

Conclusions: Aprotinin significantly reduces blood loss and the need for blood component transfusion in patients undergoing total arterial grafting without increasing the risk of myocardial injury. Aprotinin should be considered routinely in patients undergoing total arterial grafting but cautiously in patients with an elevated preoperative creatinine level.

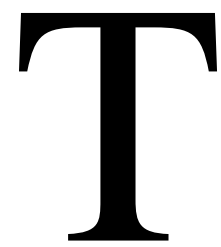

here is a growing trend toward complete arterial revascularization in patients undergoing coronary artery bypass grafting (CABG). The clinical and survival benefits of a single internal thoracic artery (SITA) graft are well recognized, and current studies suggest additional benefits of bilateral ITA (BITA) grafts. ${ }^{1}$ In a meta-analysis of the best observational studies of SITA and BITA grafts, we recently reported that BITA grafts offer survival advantages over SITA grafts. ${ }^{2}$ For total arterial grafting (TAG), the radial artery is now commonly used as the third arterial graft. $^{3}$ 
It is the principal investigator's standard practice to perform CABG using BITA and radial artery grafts. However, in my experience (D.P.T.), ${ }^{4}$ the use of BITA grafts increases mean postoperative blood loss by approximately $400 \mathrm{~mL}$ in comparison with the use of a SITA graft (mean [SD] BITA vs SITA: $1324 \mathrm{~mL}$ [362 mL] vs $945 \mathrm{~mL}$ [330 mL]; $P<$ $.01)$. This is consistent with an increased surface area of chest wall dissection when harvesting BITA vessels.

Aprotinin is a naturally occurring serine protease inhibitor that has potent hemostatic properties when used in cardiac surgery. Recent advances in the understanding of the basic mechanism of aprotinin have shown that aprotinin may in fact also have antithrombotic properties by its selective blockade of protease-activated receptor- 1 thrombin receptors. ${ }^{5}$

The major concern over the routine use of aprotinin is, however, an increased risk of graft occlusion and perioperative myocardial infarction (MI) through prothrombotic mechanisms. The International Multicenter Aprotinin Graft Patency Experience (IMAGE) trial was undertaken to resolve conflicting conclusions of studies that documented an increased risk of vein graft occlusion ${ }^{6-8}$ and those that did not. ${ }^{9}$ The IMAGE trial was conducted in 13 centers in the United States and Europe, randomizing 870 patients to aprotinin or placebo and assessing the graft patency using graft angiography. ${ }^{10}$ Unfortunately, whereas vein graft occlusion rates were similar in the 2 American groups (9\%), they differed in the European groups (23\% in the aprotinin group vs $12 \%$ in the placebo group). Even after adjustment for risk factors, the IMAGE trial suggested that aprotinin led to a slightly increased risk of venous graft occlusion when evaluated on per-patient or per-graft analysis.

We hypothesized that aprotinin would be efficacious in reducing blood loss in patients undergoing TAG without increasing the risk of myocardial injury, particularly because arterial grafts are inherently more resistant to thrombosis than vein grafts. Consequently, we performed a randomized trial of aprotinin in patients undergoing TAG to determine blood loss and need for blood products. The incidence of perioperative MI and myocardial injury was assessed by serial electrocardiograms and measurements of cardiac troponin $\mathrm{T}$ (cTnT) and creatine kinase (CK)-MB. Renal injury was assessed by serial measurement of serum creatinine.

\section{Methods \\ Ethics}

The Central Oxford Research Ethics Committee approved the trial (C00.019), which was conducted in accordance with Good Clinical Practice for Trials on Medicinal Products in the European Community (July 1990). To ensure compliance with these guidelines, the study was monitored and open to audit by Bayer and an independent body.

\section{Patient Information and Consent}

Consenting patients, fulfilling the inclusion and violating none of the exclusion criteria, provided written informed consent before participation.

\section{Power Calculations}

The sample size calculation was based on the primary efficacy variable and postoperative blood loss, and aimed to detect a difference between the aprotinin and placebo groups. From previous studies, blood loss seemed to have a log-normal distribution, so calculations were based on the geometric mean and SD. A placebo geometric mean of $1223.6 \mathrm{~mL}$ and geometric SD of 1.5 $\mathrm{mL}$ was assumed. Converting this to loge scale, this became 7.110 $\mathrm{mL}$ and $0.432 \mathrm{~mL}$, respectively. The power was set at $90 \%$, and a 2-sided significance level was set at $5 \%$, with a clinically relevant difference of $30 \%$ reduction in the geometric mean postoperative blood loss in the active group. Thirty-two evaluable patients were therefore required in each treatment group. Allowing for an invalidity rate of $15 \%$, a total sample size of 75 patients was required.

\section{Patients}

Eligible patients were those undergoing first-time isolated CABG who required at least 2 bypass grafts in which it was planned that revascularization would be performed only using arterial conduits. Patients could be undergoing elective or urgent, but not emergency (ie, immediate), surgery. Exclusion criteria were significantly impaired left ventricular function (ejection fraction estimated $<25 \%$ ), acute MI within 6 weeks, known or suspected allergy to aprotinin, possible previous exposure to aprotinin, coagulopathy or history of any bleeding diathesis, and use of any investigational drug within the previous 30 days. Patients could be withdrawn at any time for medical reasons, for example, excessive postoperative bleeding necessitating resternotomy. For patients who prematurely discontinued participation in the trial, a final assessment was completed, wherever possible, according to the schedule of the 120-hour visit.

\section{Overall Study Design and Plan}

The study was a single-center, double-blind, parallel group, placebo-controlled comparison of patients undergoing total arterial revascularization conducted by a single surgeon.

\section{Randomization}

A predetermined randomization scheme was generated by the Biometry Department of Bayer, using a fixed block size. Patients were risk stratified as elective or urgent. Sealed code break cards were available for clinical reasons.

\section{Drug Dose}

Patients were randomized 1 day before surgery to receive placebo or full-dose ("Hammersmith") aprotinin infusion, commenced after induction of anesthesia. After induction of anesthesia, and before sternotomy, a test dose $(5.0 \mathrm{~mL}$ of $1.4 \mathrm{mg} / \mathrm{mL}$ or 10,000 kallikrein inactivation unit $/ \mathrm{mL}$ ) of aprotinin or placebo solution was administered over several minutes through the central line. If there was no evidence of hypersensitivity after 10 minutes, the remaining $195 \mathrm{~mL}$ of the loading dose was administered over 20 to 30 minutes using an infusion pump. After completion of the 
loading dose, a maintenance infusion of $50 \mathrm{~mL} / \mathrm{h}$ was continued for 4 hours. A further $200 \mathrm{~mL}$ was added to the pump prime of the bypass circuit.

\section{Anesthetic Protocol}

Patients were premedicated with papaveretum and hyoscine. Anesthesia was induced using a combination of fentanyl and etomidate and maintained with nitrous oxide/air and halothane or isoflurane. Anesthesia was maintained on cardiopulmonary bypass (CPB) with a propofol infusion. Antibiotic prophylaxis was gentamicin and flucloxacillin or cefuroxime.

\section{Cardiopulmonary Bypass}

The bypass circuit was primed with 1.5 L Hartmann's solution, and a membrane oxygenator was used. Flow rates were maintained to achieve $2.4 \mathrm{~L} / \mathrm{m}^{2}$, and the patient's temperature allowed to decrease to $34^{\circ} \mathrm{C}$. Continuous flow and alpha-stat control of acid base balance was used. Perfusion pressures were maintained between 40 and $90 \mathrm{~mm} \mathrm{Hg}$, with vasoconstrictor or vasodilator agents as necessary.

\section{Anticoagulation on Bypass}

Prolongation of the activated clotting time (ACT) by aprotinin can lead to an overestimation of the degree of anticoagulation, leading to inadequate anticoagulation. Consequently, a kaolin-activated ACT greater than 600 seconds of heparin was obtained before institution of CPB with a loading dose of $300 \mathrm{U} / \mathrm{kg}$ of heparin. The ACT was rechecked periodically on CPB, and further heparin was administered if the ACT decreased less than 600 seconds. Heparin was reversed with $1 \mathrm{mg}$ of protamine per 100 units of heparin, and additional protamine was administered depending on the measured ACT.

\section{Transfusion Policy}

Doctors and nurses, independent of the trial, determined the routine postoperative management of the trial patients. If any patient experienced excessive postoperative bleeding (blood loss of $>200 \mathrm{~mL}$ in the first 2 consecutive hours in patients who were otherwise stable and in whom the bleeding was expected to stop without need for rethoracotomy), then, at the discretion of the investigator, double-blind medication was stopped, and an open-label aprotinin infusion started according to routine clinical practice.

In general terms, hemostatic factors were requested when bleeding was approximately $200 \mathrm{~mL} / \mathrm{h}$ in otherwise stable patients. During the preoperative and postoperative periods, blood was transfused if the hemoglobin decreased to less than or was equal to $9 \mathrm{~g} / \mathrm{dL}$. Further units were administered to ensure that hemoglobin was maintained greater than $9 \mathrm{~g} / \mathrm{dL}$.

\section{Analysis}

The study was analyzed on an intention-to-treat (ITT) basis and included those patients who received open-label aprotinin. All patients receiving trial treatment were included in the safety analysis.

\section{Trial Objectives}

The primary aim of the trial was to assess the efficacy of aprotinin in reducing postoperative blood loss after TAG and the need for transfusion of blood and blood products. In addition, the frequency of resternotomy, time to extubation, and total duration of hospital stay was recorded. Myocardial injury was assessed by serial electrocardiographic changes and serum CK-MB and cTnT (calculated as an area under the curve [AUC] from serial measurements) and renal function by measurement of serum creatinine.

\section{Statistical Analyses}

Summary statistics for the demographic data include the arithmetic mean and SD (normally distributed data), the geometric mean and SD (log-normally distributed data), and the median and range (non-normal data) or frequency counts (categoric data, in which the number of categories were small).

Treatment groups were compared at baseline to check for balance in prognostic factors using the Student 2-sample $t$ test (normally distributed variables), Wilcoxon 2-sample test (nonnormally distributed variables), and Fisher's exact test (categoric variables).

The primary efficacy parameter, postoperative blood loss, was tested for normality using the Shapiro-Wilk test, and because it was approximately normally distributed after applying log transformation, the treatment groups were compared using the Student 2-sample $t$ test.

Because the number of units of donor blood and other blood products and the frequency of resternotomy were categoric, nonnormal data, the Wilcoxon 2-sample test was used to compare treatment groups, and the Cochrane-Mantel-Haenszel test was used when the number of categories was small. The number of patients receiving a blood transfusion at any time during the study was summarized using frequency counts and percentages, and treatment groups were compared using Fisher's exact test.

For cTnT and CK-MB, the AUC was computed for each patient. By providing an integrated value for the release of a chemical or metabolite over a given time frame, the AUC increases the chances of detecting minor differences between 2 groups while minimizing the chances of a false-positive finding by making multiple comparisons of individual time points. Furthermore, the AUC allows comparison of the data from all patients in a trial, including those with minor elevations in any chemical or metabolite, even when they remain within the normal range.

The AUC was calculated over 8 time points from the preoperation baseline ( 0 hours) to 123 hours after baseline by using the trapezoidal rule with interpolation for missing intermediate time points and then dividing by 123 to give units in nanograms per milliliter. Where a measurement at the final time point was missing, the AUC was calculated over the time points present and then divided by the appropriate time interval to give units in nanograms per milliliter. The AUC data for cTnT and CK-MB were compared between the 2 treatment groups using the Wilcoxon 2-sample test.

\section{Results}

Patients

Between April 2000 and April 2001, 74 patients in total were randomized in this double-blind, parallel group, placebo-controlled, single-center study (Table 1). Seventy 
TABLE 1. Withdrawals and protocol deviations in intention-to-treat analyses

\begin{tabular}{|c|c|c|c|}
\hline & & Placebo & Aprotinin \\
\hline Randomization & & 37 & 37 \\
\hline \multirow[t]{3}{*}{ Withdrawal } & No medication & 1 & 1 \\
\hline & Randomization code broken & 1 & \\
\hline & Randomization code broken and disallowed medication & 1 & \\
\hline Included in intention-to-treat analyses & & 34 & 36 \\
\hline \multirow[t]{6}{*}{ Protocol deviations } & Received venous conduit & 1 & 1 \\
\hline & Received open-label aprotinin & 5 & 1 \\
\hline & Venous conduit and open-label aprotinin & 1 & 1 \\
\hline & Coagulopathic drug and open-label aprotinin & 1 & \\
\hline & Venous conduit, coagulopathic drug, open-label aprotinin & 1 & \\
\hline & Received anticoagulant drug preoperative & 2 & 2 \\
\hline
\end{tabular}

TABLE 2. Summary of demographic data

\begin{tabular}{lccc}
\hline & Placebo & Aprotinin & $\boldsymbol{P}$ \\
\hline Age (mean, SD) & $61(8)$ & $60(8)$ & .6 \\
Percent male & $94 \%$ & $92 \%$ & 1.00 \\
Height in centimeters (mean, SD) & $174(8)$ & $171(8)$ & .06 \\
Weight in kilograms (mean, SD) & $83(11)$ & $83(12)$ & .9 \\
Diabetes mellitus (types I and II) & $5(14 \%)$ & $6(16 \%)$ & .9 \\
Percent elective admission & $68 \%$ & $67 \%$ & 1.00 \\
Percent urgent admission & $32 \%$ & $33 \%$ & \\
\hline
\end{tabular}

TABLE 3. Summary of intraoperative data

\begin{tabular}{|c|c|c|c|c|}
\hline & & Placebo & Aprotinin & $P$ \\
\hline Number & & 34 & 36 & \\
\hline $\begin{array}{l}\text { Duration of operation in } \\
\text { hours (mean, SD) }\end{array}$ & & $3.1(0.5)$ & $3.1(0.6)$ & .7 \\
\hline $\begin{array}{l}\text { Mean number of distal } \\
\text { anastomoses }\end{array}$ & & 3.2 & 3.0 & \\
\hline $\begin{array}{l}\text { Number of distal } \\
\text { anastomoses }\end{array}$ & 2 & $4(12 \%)$ & $7(19 \%)$ & .56 \\
\hline & $\begin{array}{l}3 \\
4 \\
5\end{array}$ & $\begin{array}{c}20(59 \%) \\
9(27 \%) \\
1(3 \%)\end{array}$ & $\begin{array}{c}22(61 \%) \\
7(19 \%) \\
0\end{array}$ & \\
\hline $\begin{array}{l}\text { Duration of crossclamp } \\
\text { in minutes (mean, SD) }\end{array}$ & & $54(12)$ & $48(12)$ & .01 \\
\hline
\end{tabular}

patients were available for an analysis on an ITT basis, 34 in the placebo group and 36 in the aprotinin group (Table 1). In the placebo group, 3 patients were not available for ITT analyses ( 1 was withdrawn before receiving trial treatment, 1 had randomization code broken, and 1 received disallowed concomitant medication). In the aprotinin group, 1 patient was withdrawn before receiving trial treatment.

Protocol deviations, which did not exclude patients from the ITT analyses, are summarized in Table 1. Of particular note, open-label aprotinin was administered to 9 patients in the placebo group (26\%) and to 2 patients in the aprotinin group (6\%), and venous conduit was used in 4 patients in the placebo group (12\%) and in 2 patients in the aprotinin group (6\%).

\section{Demographic Features}

The groups were similar with respect to age, gender, height, weight, and urgency of operation (Table 2). In both groups, approximately one third of patients underwent urgent operation for unstable angina and $15 \%$ of all patients were treated for diabetes.

\section{Intraoperative Data}

The groups were similar with respect to overall operative time and number of distal anastomoses (Table 3). Nevertheless, the mean crossclamp time was 6 minutes longer in the placebo group compared with the aprotinin group (54 [12] vs 48 [12] minutes, respectively; $P=.01$ ).

\section{Postoperative Blood Loss and Transfusion Requirements}

There was a highly significant reduction in the median (interquartile range) blood loss in the aprotinin group compared with the placebo group (785 [590-1025] vs 1525 [1175-1920], respectively) (Table 4). One reason for an apparently high blood loss in the placebo group is that one third of these patients were urgent cases, and many would have been taking aspirin and clopidogrel. The need for red blood cell transfusion was reduced from $77 \%$ in the placebo group to $39 \%$ in the aprotinin group $(P=.0001)$. Overall, the mean number of transfused red blood cell units was 0.8 in the aprotinin group and 2.6 in the placebo group. Similarly, there was a highly significant reduction in the need for coagulation products in the aprotinin group. A single patient $(3 \%)$ in the aprotinin group required coagulant products in contrast with $24 \%$ of patients in the placebo group.

\section{Secondary Clinical Outcomes}

One patient in each group underwent reexploration for bleeding. There was no difference in time to extubation or time to drain removal in the 2 groups (Table 5). The median 
TABLE 4. Postoperative blood loss and transfusion requirements

\begin{tabular}{|c|c|c|c|c|}
\hline & & Placebo (34) & Aprotinin (36) & $\boldsymbol{P}$ \\
\hline \multicolumn{2}{|c|}{$\begin{array}{l}\text { Total postoperative blood loss in milliliters } \\
\text { (median and interquartile range) }\end{array}$} & $1525(1175-1920) \mathrm{mL}$ & $785(590-1025) \mathrm{mL}$ & $<.0001$ \\
\hline \multicolumn{5}{|c|}{ Requirement for red blood cell transfusion } \\
\hline \multicolumn{2}{|c|}{ Percent of patients requiring blood transfusion } & $77 \%$ & $39 \%$ & $=.0001$ \\
\hline \multirow[t]{4}{*}{ Number of transfused units } & 0 & $8(24 \%)$ & $22(61 \%)$ & \\
\hline & 1 & $5(15 \%)$ & $7(19 \%)$ & \\
\hline & $2-4$ & $15(44 \%)$ & $6(17 \%)$ & \\
\hline & 5 or $>$ & $6(18 \%)$ & $1(3 \%)$ & \\
\hline \multicolumn{5}{|c|}{ Requirement for coagulant products } \\
\hline \multicolumn{2}{|c|}{ Fresh frozen plasma } & $8(24 \%)$ & $1(3 \%)$ & \\
\hline \multicolumn{2}{|l|}{ Platelets } & $7(21 \%)$ & $1(3 \%)$ & \\
\hline \multicolumn{2}{|l|}{ Cryoprecipitate } & $1(3 \%)$ & 0 & \\
\hline
\end{tabular}

duration of high dependency unit and postoperative hospital stay was 1 and 6 days, respectively, in both groups.

\section{Biochemical Data}

Serial data for $\mathrm{cTnT}$ and CK-MB release are presented in Figures 1 and 2. cTnT and CK-MB AUCs were calculated over 8 time points from preoperation baseline ( 0 hours) to the fifth postoperative day (123 hours) dividing by 123 to give units in nanograms per milliliter. For cTnT, the respective AUC (median and IQ range) was $0.15(0.08-0.22)$ in the placebo group and $0.12(0.08-0.18)$ in the aprotinin group $(P$ $=.40$ ). For CK-MB, the respective AUC (median and IQ range) was 6.0 (2.4-12.8) in the placebo group and 4.8 (3.5-8.2) in the aprotinin group $(P=.64)$.

\section{Electrocardiographic Criteria}

Perioperative MI (defined as the appearance of new q waves or loss of $>20 \% \mathrm{R}$ waves with cTnT $>2.0 \mathrm{ng} / \mathrm{mL}$ and CK-MB $>100 \mathrm{ng} / \mathrm{mL}$ ) occurred in 2 patients (3\%; 1 in each group).

\section{Renal Injury}

Serial changes in serum creatinine in both groups are presented in Figure 3. Overall, there was no significant difference between the groups. There were, however, 4 patients in the aprotinin group and 2 patients in the control group whose postoperative creatinine exceeded $2 \mathrm{mg} / \mathrm{L}$ on day 5 . Of particular interest, 3 of these 4 patients in the aprotinin group had elevated preoperative creatinine levels (1.7, 1.7, $1.5 \mathrm{mg} / \mathrm{L}$, respectively), whereas both the control patients had normal preoperative creatinine values. In addition, the 4 patients in the aprotinin group had other complications that may have contributed to renal impairment, namely, longstanding type I diabetes mellitus, severe prolonged postoperative ileus, perioperative MI, and "prophylactic" intraaortic balloon pump insertion for severely impaired left ventricle function. In the control group, 1 patient had no obvious explanation for a precipitous increase in postoper-
TABLE 5. Secondary clinical outcomes

\begin{tabular}{lccr}
\hline & Placebo & Aprotinin & $\boldsymbol{P}$ \\
\hline $\begin{array}{l}\text { Number } \\
\text { Reexploration for bleeding }\end{array}$ & $1(3 \%$ & 36 & \\
$\begin{array}{l}\text { Hours to extubation in hours } \\
\text { (median, 95\% CI) }\end{array}$ & $8(6-27)$ & $1(3 \%)$ & 1.00 \\
$\begin{array}{l}\text { Hours to thoracic drains } \\
\quad \text { removal (median, 95\% CI) }\end{array}$ & $29(27-41)$ & $26(22-28)$ & .34 \\
$\begin{array}{l}\text { Days in high dependency unit } \\
\quad \text { (median, I0 range) }\end{array}$ & $1(0.9-1.2)$ & $1(0.9-1.1)$ & .06 \\
$\begin{array}{l}\text { Postoperative days in hospital } \\
\text { (median, I0 range) }\end{array}$ & $6(6-7)$ & $6(6-7)$ & .84 \\
\hline
\end{tabular}

$\mathrm{Cl}$, Confidence interval.

ative creatinine, whereas the other patient had a hypovolemic cardiac arrest when a clip on the stump of the proximal right ITA eroded into the subclavian artery requiring emergency reexploration.

\section{Discussion}

There is growing evidence that TAG, and in particular the use of BITA grafts, offers survival and other clinical advantages over a single ITA graft and supplemental vein grafts. ${ }^{1,2}$ Inasmuch as we had previously reported that the use of BITA grafts increases postoperative blood loss, the routine use of aprotinin seemed persuasive, especially because arterial grafts are inherently more resistant to thrombosis than vein grafts. To our knowledge, this is the first randomized trial of aprotinin in patients undergoing TAG.

\section{Potential Limitations of this Trial}

Although this was a trial of TAG, 4 patients in the placebo group (12\%) and 2 patients in the aprotinin group (6\%) received additional vein grafts. This reflected our "learning" phase with TAG and now occurs in less than $5 \%$ of our patients. In any event, it did not affect the results of the trial when analyzed on an ITT basis. 


\section{Blood Loss, Blood Transfusion, and Use of Coagulation Products}

This trial demonstrates that aprotinin results in a dramatic reduction in blood loss and blood product requirements in patients undergoing TAG. Aprotinin almost halves blood loss, halves the number of patients requiring red blood cell transfusion, and significantly reduces the number of units transfused per patient and the need for coagulant products. Even so, our results may still underestimate the benefits of aprotinin; in the ITT analyses, $26 \%$ of patients in the placebo group received open-label aprotinin compared with $6 \%$ of patients in the aprotinin group.

\section{Aprotinin and Risk of Myocardial Injury}

The overall AUC for both troponin and CK-MB release, reflecting a global index of myocardial injury, was lower in the aprotinin group, although this did not reach statistical significance.

There is conflicting evidence as to whether aprotinin increases the risk of perioperative myocardial injury in conventional CABG using an ITA and vein grafts. Cosgrove and colleagues ${ }^{6}$ reported an increased incidence of perioperative $\mathrm{MI}$ in patients undergoing redo $\mathrm{CABG}$ from $7 \%$ of patients $(4 / 56)$ in the placebo group to $16 \%$ of patients (9/57) receiving high-dose aprotinin. Lemmer and coworkers $^{7}$ also reported an increased risk of perioperative MI in patients receiving high-dose aprotinin $(8.9 \%$ vs $5.6 \%)$, although this did not reach statistical significance. In contrast, the IMAGE study assessed 831 patients' electrocardiograms and cardiac enzymes and reported that aprotinin did not increase the risk of MI compared with the placebo group. ${ }^{10}$ Similarly, a multicenter study of redo CABG by Alderman and colleagues ${ }^{11}$ showed no difference in the incidence of MI in the placebo or aprotinin-treated groups (29\%). These conflicting observations may be explained, in part, by the meta-analysis of 72 trials involving more than 8000 patients performed by Levi and colleagues. ${ }^{12}$ They reported that high-dose, but not low-dose, aprotinin increased the risk of perioperative MI.

It must be emphasized that these studies did not include patients undergoing TAG. Consequently, a secondary aim of our trial was to assess the effect of aprotinin on both MI and global injury. Perioperative MI (suggesting focal injury) was determined by a combination of serial electrocardiograms and cardiac-specific enzymes (cTnT, CK-MB), whereas global myocardial ischemia was determined from an integrated AUC for total enzyme release calculated from serial enzyme measurements at 8 time points over 5 days.

Our trial demonstrates that aprotinin does not increase the risk of perioperative myocardial injury in the setting of TAG. It is notable, however, that only 2 patients (3\%) experienced a perioperative MI, according to electrocardio- graphic and biochemical criteria. Because most perioperative MIs are caused by acute graft failure, ${ }^{8}$ it is possible that this may represent a reduced risk of acute failure of arterial conduits in comparison with vein grafts. This is certainly consistent with angiographic data from several studies reporting significantly better patency of arterial conduits in comparison with vein grafts early after $\mathrm{CABG},{ }^{13}$ but in the absence of our own angiographic data this must remain speculative.

This is also consistent with various animal studies that have shown aprotinin to be cardioprotective particularly when used as a cardioplegia additive.

\section{Aprotinin and Risk of Renal Injury}

Although several studies have reported that aprotinin does not increase the risk of renal failure after cardiac surgery, ${ }^{14}$ others have documented an increase in postoperative serum creatinine levels. In a multicenter, randomized study of 212 patients undergoing valve replacement, postoperative increases in creatinine exceeding $0.5 \mathrm{mg} / \mathrm{dL}$ occurred in $30 \%$, $14 \%$, and $8 \%$ of the high-dose aprotinin, low-dose aprotinin, and placebo groups, respectively, and were statistically significantly different. ${ }^{15}$ In a randomized study by Cosgrove and associates, ${ }^{6}$ postoperative increases in serum creatinine of greater than $0.5 \mathrm{mg} / \mathrm{dL}$ occurred in $25 \%, 20 \%$, and $18 \%$ in the high-dose aprotinin, low-dose aprotinin, and placebo groups, respectively, although these differences were not statistically significant. Similarly, a multicenter, randomized, controlled trial showed a modest postoperative increase in serum creatinine levels in $19 \%$ of the patients (20/108) in the aprotinin group compared with $12 \%$ of the patients (13/108) in the placebo group, although this was not statistically significant. ${ }^{14}$

Overall, as judged by median and interquartile ranges, our trial did not show any adverse effect of aprotinin on renal function as judged by serial serum creatinine levels. This is consistent with the recent trial by Schweizer and colleagues, ${ }^{16}$ who randomized 57 patients undergoing cardiac surgery with CPB to high-dose aprotinin and placebo and detected no difference in several sophisticated parameters of renal function between the groups. It should be noted, however, that 4 patients in the aprotinin group and 2 patients in the control group had postoperative creatinine levels exceeding $2 \mathrm{mg} / \mathrm{L}$ on day 5 . In the control group, 1 patient had a hypovolemic cardiac arrest requiring emergency thoracotomy to control bleeding, and the other patient had no obvious explanation for the precipitous increase in creatinine. Of the 4 patients in the aprotinin group, $3 \mathrm{had}$ elevated preoperative creatinine levels, and 4 had other complications that may have contributed to postoperative renal impairment. It is therefore uncertain whether it was the elevated preoperative creatinine level or a perioperative complication that contributed most to the postoperative 


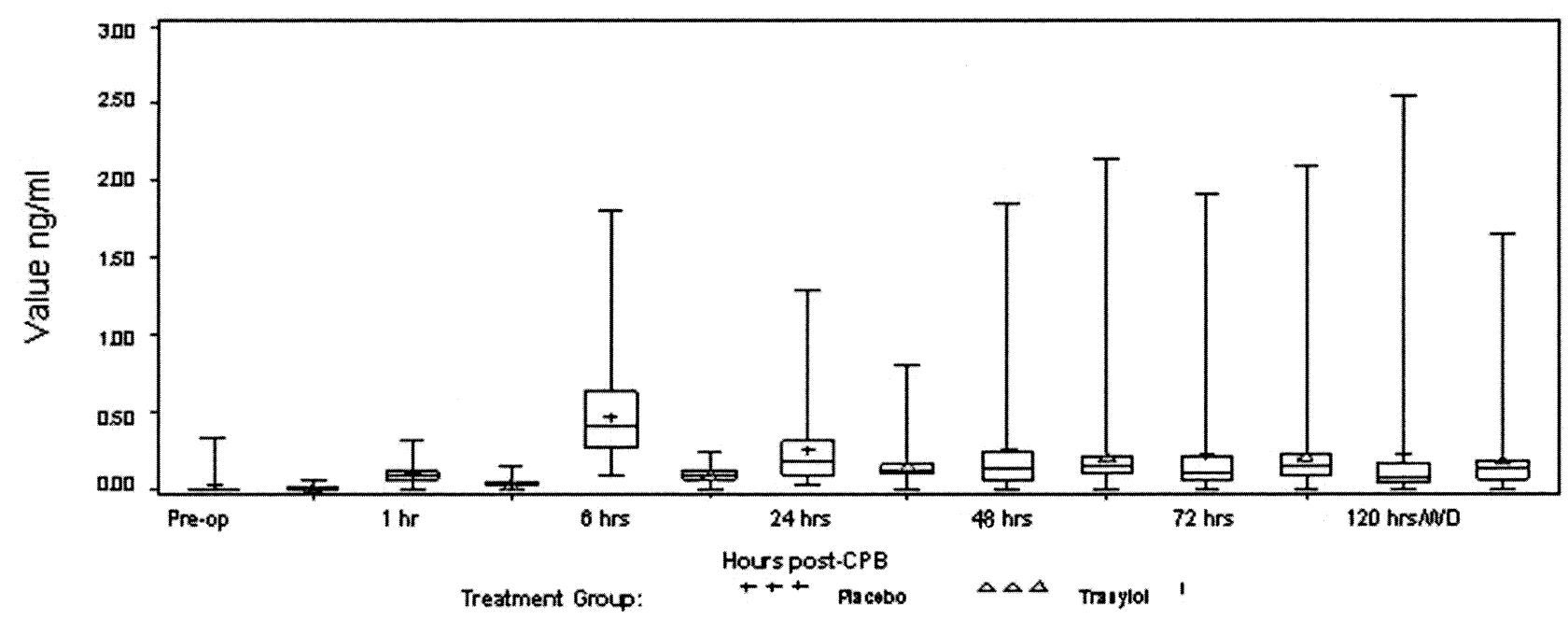

Figure 1. TnT in the 2 groups (median and interquartile range [box]; spread of the data [whiskers]).

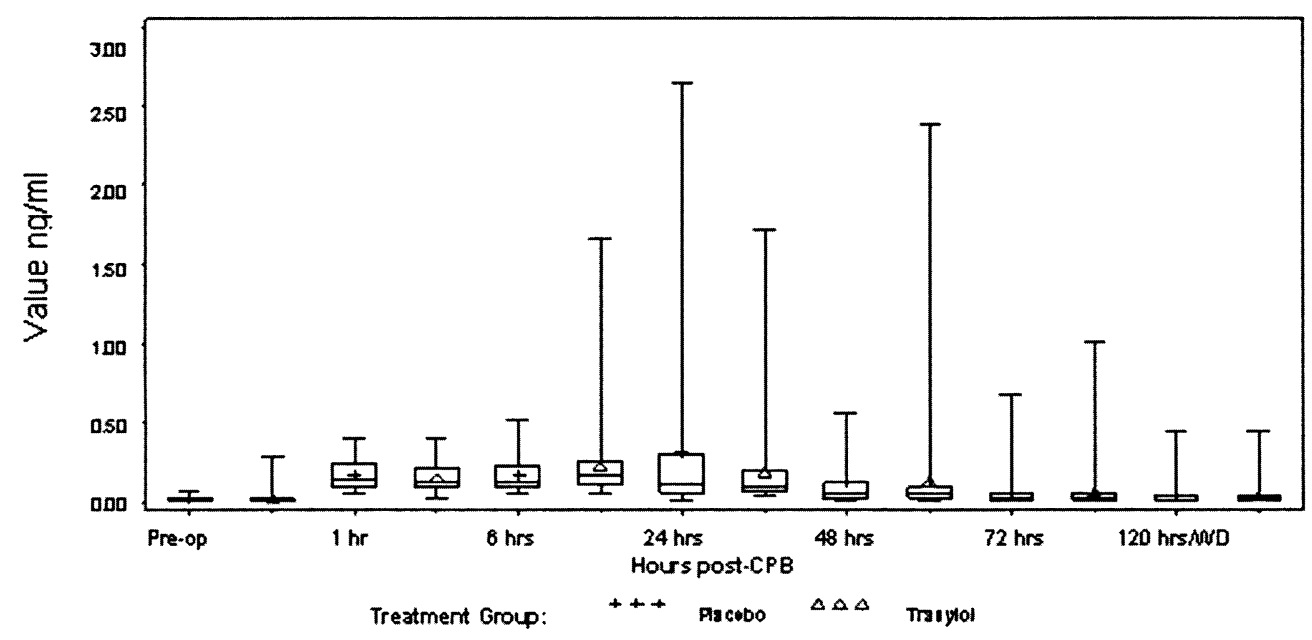

Figure 2. CK-MB in the 2 groups (median and interquartile range [box]; spread of the data [whiskers]).

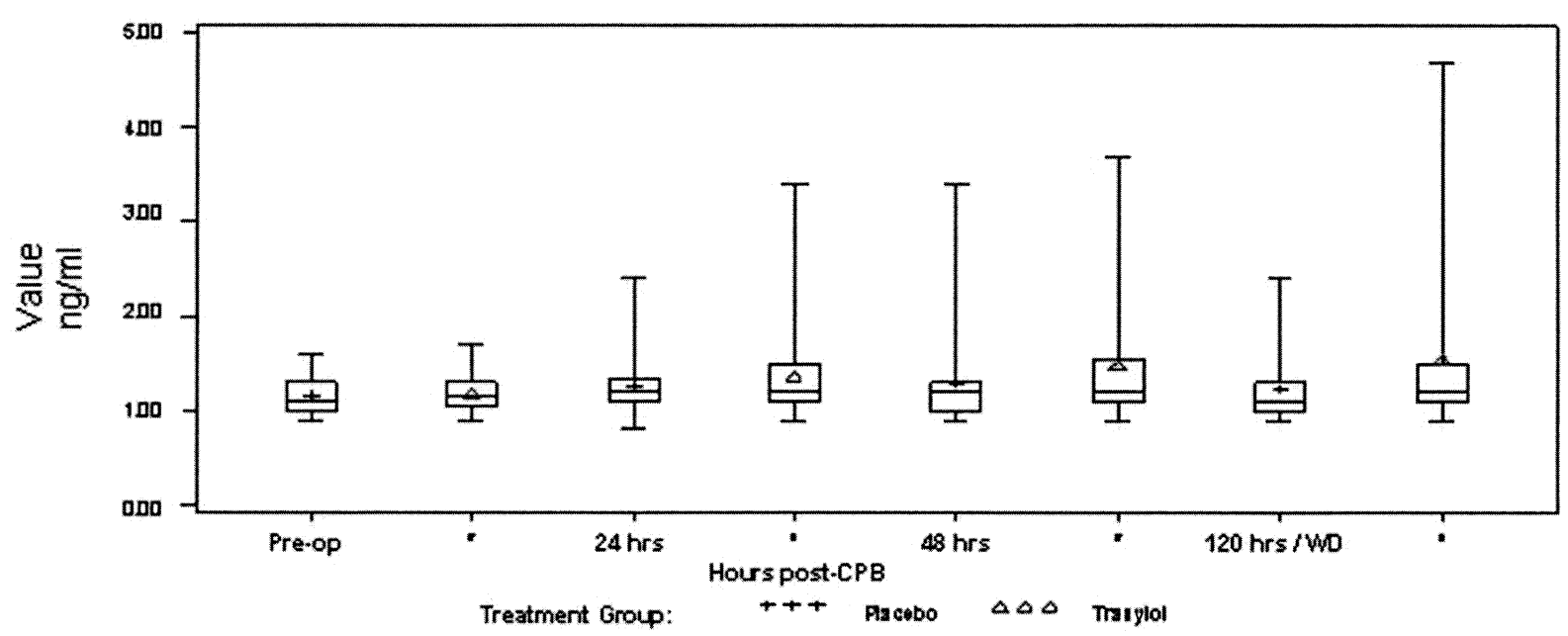

Figure 3. Serum creatinine in the 2 groups (median and interquartile range [box]; spread of the data [whiskers]). 
elevation, but it would seem sensible to use aprotinin cautiously in those with preoperative renal impairment.

\section{Conclusion}

This trial demonstrates that aprotinin results in a dramatic reduction in blood loss and blood product requirements in patients undergoing TAG without increasing the risk of myocardial injury. Aprotinin should be used more cautiously in those with preoperative renal impairment.

D.T. conceived the study, designed the protocol, performed all the operations, and wrote the main draft of the article. V.D. performed literature searches and assisted in drafting the manuscript. M.N. assisted in the daily conduct of the study as well as collecting blood samples. A.D. enrolled patients and was responsible for drug administration and collection of blood samples. Dr Mark Summery and Dr Edward Tucker provided particular assistance in the design of the protocol and in ensuring the smooth running of the trial.

\section{References}

1. Lytle BW, Blackstone EH, Loop FD, Houghtaling PL, Arnold JH, Akhrass R, et al. Two internal thoracic artery grafts are better than one. J Thorac Cardiovasc Surg. 1999;117:855-72.

2. Taggart DP, D'Amico R, Altman DG. Effect of arterial revascularisation on survival: a systematic review of studies comparing bilateral and single internal mammary arteries. Lancet. 2001;358:870-5.

3. Taggart DP. The radial artery as a conduit for coronary artery bypass grafting. Heart. 1999;82:409-10.

4. Taggart DP. The effects of a platelet activating factor antagonist on the respiratory, myocardial and cerebral consequences of cardiopulmonary bypass and further observations on cardiac surgery without cardiopulmonary bypass, in Department of Bioengineering. Glasgow, UK: Strathclyde; 2000.

5. Landis RC, Asimakopoulos G, Poullis M, Thompson R, Nourshargh S, Haskard DO, et al. Effect of aprotinin (Trasylol) on the inflammatory and thrombotic complications of conventional cardiopulmonary bypass surgery. Heart Surg Forum. 2001;4(Suppl 1):S35-9.
6. Cosgrove DM 3rd, Heric B, Lytle BW, Taylor PC, Novoa R, Golding LA, et al. Aprotinin therapy for reoperative myocardial revascularization: a placebo-controlled study. Ann Thorac Surg. 1992;54:1031-8.

7. Lemmer JH Jr, Stanford W, Bonney SL, Breen JF, Chomka EV, Eldredge WJ, et al. Aprotinin for coronary bypass operations: efficacy, safety, and influence on early saphenous vein graft patency. A multicenter, randomized, double-blind, placebo-controlled study. J Thorac Cardiovasc Surg. 1994;107:543-53.

8. van der Meer J, Hillege HL, Ascoop CA, Dunselman PH, Mulder BJ, van Ommen GV, et al. Aprotinin in aortocoronary bypass surgery: increased risk of vein-graft occlusion and myocardial infarction? Supportive evidence from a retrospective study. Thromb Haemost. 1996; 75:1-3.

9. Havel M, Grabenwoger F, Schneider J, Laufer G, Wollenek G, Owen A, et al. Aprotinin does not decrease early graft patency after coronary artery bypass grafting despite reducing postoperative bleeding and use of donated blood. J Thorac Cardiovasc Surg. 1994;107:807-10.

10. Alderman EL, Levy JH, Rich JB, Nili M, Vidne B, Schaff H, et al., Analyses of coronary graft patency after aprotinin use: results from the International Multicenter Aprotinin Graft Patency Experience (IMAGE) trial. J Thorac Cardiovasc Surg. 1998;116:716-30.

11. Alderman EL, Levy JH, Rich JB, Nili M, Vidne B, Schaff H, et al. A multicenter, double-blind, placebo-controlled trial of aprotinin for reducing blood loss and the requirement for donor-blood transfusion in patients undergoing repeat coronary artery bypass grafting. Circulation. 1995;92:2236-44.

12. Levi M, Cromheecke ME, de Jonge E, Prins MH, de Mol BJ, Briet E, et al. Pharmacological strategies to decrease excessive blood loss in cardiac surgery: a meta-analysis of clinically relevant endpoints. Lancet. 1999;354:1940-7.

13. Endo M, Nishida H, Tomizawa Y, Kasanuki H. Benefit of bilateral over single internal mammary artery grafts for multiple coronary artery bypass grafting. Circulation. 2001;104:2164-70.

14. Lemmer JH Jr, Stanford W, Bonney SL, Chomka EV, Karp RB, Laub $\mathrm{GW}$, et al. Aprotinin for coronary artery bypass grafting: effect on postoperative renal function. Ann Thorac Surg. 1995;59:132-6.

15. D'Ambra MN, Akins CW, Blackstone EH, Bonney SL, Cohn LH, Cosgrove DM, et al. Aprotinin in primary valve replacement and reconstruction: a multicenter, double-blind, placebo-controlled trial. J Thorac Cardiovasc Surg. 1996;112:1081-9.

16. Schweizer A, Hohn L, Morel DR, Kalangos A, Licker M. Aprotinin does not impair renal haemodynamics and function after cardiac surgery. Br J Anaesth. 2000;84:16-22. 